\title{
Quantized vortices in an exciton-polariton condensate
}

\author{
K. G. LAGOUDAKIS ${ }^{1 *}$, M. WOUTERS ${ }^{2 * \dagger}$, M. RICHARD ${ }^{1}$, A. BAAS ${ }^{1}$, I. CARUSOTTO ${ }^{3}$, R. ANDRÉ ${ }^{4}$, \\ LE SI DANG4 AND B. DEVEAUD-PLÉDRAN ${ }^{1}$
}

\author{
${ }^{1}$ IPEQ, Ecole Polytechnique Fédérale de Lausanne(EPFL), Station 3, 1015 Lausanne, Switzerland \\ ${ }^{2}$ ITP, Ecole Polytechnique Fédérale de Lausanne(EPFL), Station 3, 1015 Lausanne, Switzerland \\ ${ }^{3}$ INFM-CNR BEC and Dipartimento di Fisica, Universita di Trento, via Sommarive 14, 38050 Povo (Trento), Italy \\ ${ }^{4}$ Institut Néel, CNRS, 25 Avenue des Martyrs, 38042 Grenoble, France \\ ${ }^{\dagger}$ Previously: TFVS, Universiteit Antwerpen, Universiteitsplein 1, 2610 Antwerpen, Belgium \\ *e-mail: konstantinos.lagoudakis@epfl.ch; michiel.wouters@epfl.ch
}

One of the most striking quantum effects in an interacting Bose gas at low temperature is superfluidity. First observed in liquid ${ }^{4} \mathrm{He}$, this phenomenon has been intensively studied in a variety of systems for its remarkable features such as the persistence of superflows and the proliferation of quantized vortices ${ }^{1}$. The achievement of Bose-Einstein condensation in dilute atomic gases ${ }^{2}$ provided the opportunity to observe and study superfluidity in an extremely clean and well-controlled environment. In the solid state, Bose-Einstein condensation of exciton polaritons has been reported recently ${ }^{3-6}$. Polaritons are strongly interacting light-matter quasiparticles that occur naturally in semiconductor microcavities in the strong-coupling regime and constitute an interesting example of composite bosons. Here, we report the observation of spontaneous formation of pinned quantized vortices in the Bose-condensed phase of a polariton fluid. Theoretical insight into the possible origin of such vortices is presented in terms of a generalized Gross-Pitaevskii equation. Whereas the observation of quantized vortices is, in itself, not sufficient for establishing the superfluid nature of the non-equilibrium polariton condensate, it suggests parallels between our system and conventional superfluids.

Vortices in superfluids carry quantized phase winding and circulation of the superfluid particles around their core. By definition, vortices are characterized by (1) a rotation of the phase around the vortex by an integer multiple of $2 \pi$, commonly known as the topological charge of the vortex and (2) the vanishing of the superfluid population at their core. Owing to their major importance for the understanding of superfluidity, they have been intensively studied theoretically ${ }^{7}$ and experimentally ${ }^{8-10}$ in disorder-free, stirred three-dimensional Bose-Einstein condensates (BECs) of dilute atomic gases and in quasi-two-dimensional BECs where they spontaneously emerge from thermal fluctuations $s^{11,12}$ and are strictly related to the Berezinskii-Kosterlitz-Thouless phase transition $^{13-15}$. Here, we observed the spontaneous appearance of pinned singly quantized vortices as an intrinsic feature of non-equilibrium polariton BECs in the presence of disorder. The same planar CdTe microcavity sample was used as in our previous studies ${ }^{3,16,17}$. The polariton condensate was created by means of non-resonant continuous-wave optical excitation, the intensity of which is used to drive the polaritons throughout the phase transition, as demonstrated by the condensate emission energy being located close to the bottom of the polariton dispersion. The condensate steady state is determined by a dynamical balance between the incoming and the outgoing flow of polaritons: in contrast to atomic BECs, the polariton condensate is in an intrinsically non-equilibrium condition. From this point of view, it is therefore closer to a laser, but fundamental differences are still to be noted with respect to a standard photon laser: the bosonic particles under investigation are in fact polaritons rather than photons, and the accumulation of polaritons into the condensate mode stems from stimulated exciton-exciton and exciton-phonon scattering rather than electron-hole recombination. Owing to the photonic component of the cavity polariton, all of the statistical properties of the intracavity polariton field are contained in the far-field of the polariton luminescence ${ }^{18}$, thus allowing access to the phase and the amplitude of the polariton fluid order parameter. The straightforward way in which the spatial shape of the order parameter can be extracted in our microcavity polariton system is a major advantage with respect to most other quantum condensed systems. In our previous experiments ${ }^{3,16,17}$, we used cross-correlation techniques to probe the spatially resolved phase correlations of the polariton condensate. Here, we use the same technique to probe the existence of vortices and to identify the characteristic $2 \pi$ phase rotation. To visualize the phase of the macroscopic wavefunction, the condensate luminescence is superimposed to its retroreflected image. For a vortex-free polariton condensate with a spatially uniform phase, the non-zero angle between the two beams results in a system of parallel fringes. In the presence of a vortex, a pair of fork-like dislocations, symmetrically located with respect to the retroreflector axis, appear in the interferogram as a consequence of the $2 \pi$ phase rotation around the vortex core (Fig. 1a,b). Similar dislocations were observed in other optical systems ${ }^{19-21}$ as well as in atomic $\mathrm{BECs}^{10,22,23}$. In this latter case, a matter-wave interference pattern is obtained by combining the coherent matter fields originating from the expansion of two independent condensates ${ }^{24}$.

We have repeated the experiment by moving the excitation spot at different spatial positions on the sample and for different values of the excitation intensity and vortices can be identified in 

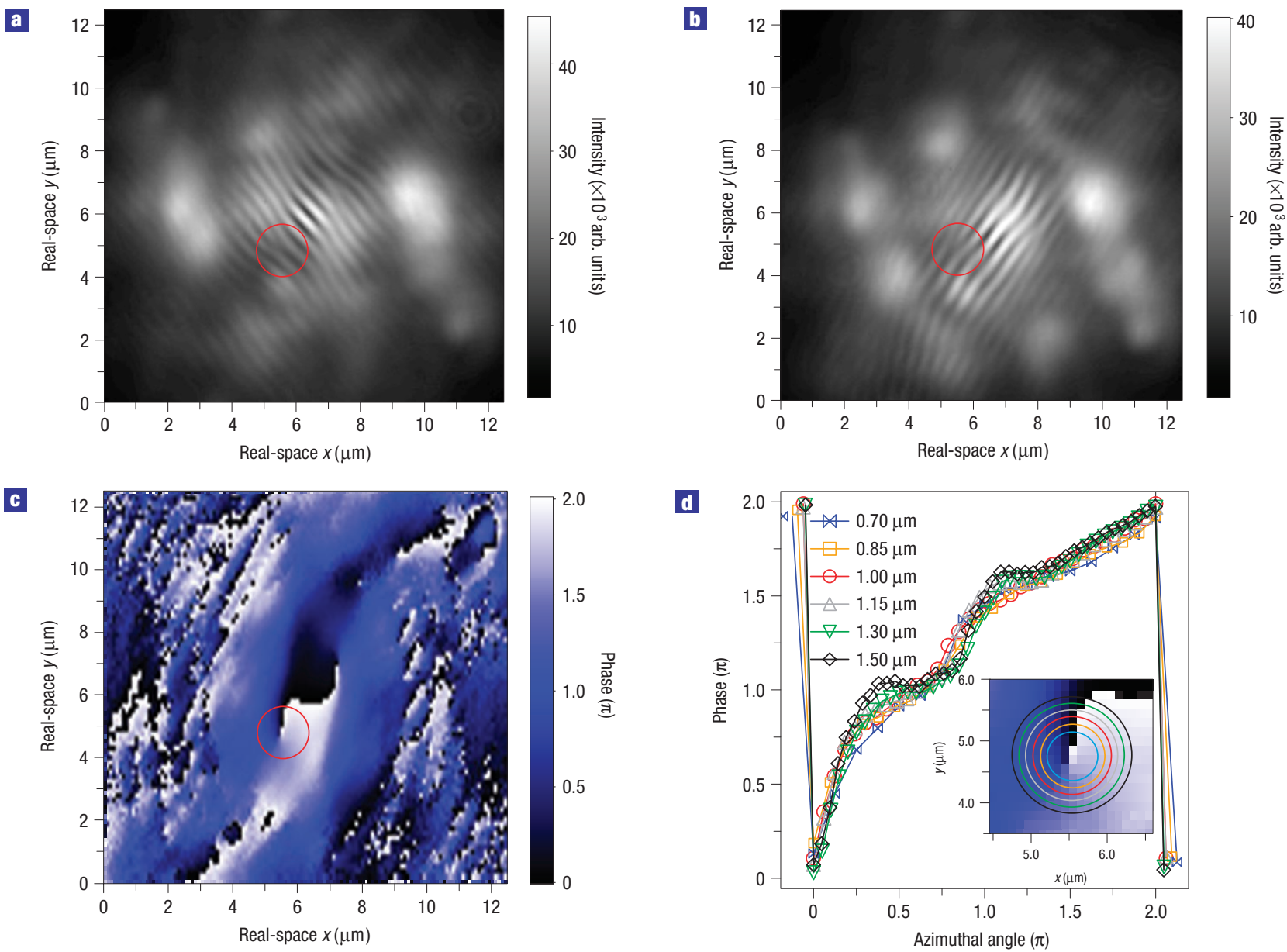

Figure 1 Interferogram and extracted phase. a, Interferogram with vortex: the fork-like dislocation can be seen in the red circle. $\mathbf{b}$, Interferogram carrying the same information, but this time the vortex is overlapped with a different region of the condensate and for a different fringe orientation. The vortex appears at the same real-space coordinates as before. $\mathbf{c}$, Real-space phase profile calculated from the interferogram in $\mathbf{a}$. The red circle encloses the vortex (same real-space area as in $\mathbf{a}, \mathbf{b})$. $\mathbf{d}$, Phase as a function of the azimuthal angle for a range of different radii as shown in the inset of $\mathbf{d}$ (magnification of $\mathbf{c}$ ). Note that the data are repeated before and after the azimuthal angles 0 and $2 \pi$ to better illustrate the $2 \pi$ shift.

a significant fraction of the interferograms, carrying in all cases a unit topological charge. This is demonstrated by the single $2 \pi$ phase winding around their core as shown in Fig. 1c,d. Note that the observed phase winding extends up to a region far from the vortex core, which makes the $2 \pi$ rotation of the phase unambiguous. The precise phase values measured along closed circulation loops of various radii around the vortex are plotted in Fig. 1d. For a single vortex in an otherwise homogeneous condensate, the phase would be a linear function of the azimuthal angle ${ }^{7}$. In the present polariton system, the phase profile is distorted by the fact that the underlying medium is disordered. To be confident that the observed dislocation is due to the phase singularity of a polariton vortex, we carried out the same interferometric measurement several times, overlapping the vortex with different areas of the condensate (without a vortex). We also changed the orientation of the interference pattern. Whatever the angle between the two interfering beams, one fork-like dislocation was always present at the vortex position. This is shown in Fig. 1b: the vortex is the same as in Fig. 1a, although here it is overlapped with a different region of the condensate and with a different overlap angle (rotated fringes). Thus, the fork-like interference pattern cannot be due to trivial optical effects such as point- or line-like structural defects of the microcavity perturbing the polariton emission wavefront. This unambiguously demonstrates the existence of a quantized phase winding that is the most peculiar property of vortices in a quantum fluid.

As we already mentioned, another key characteristic of the vortices is the absence of superfluid density in their core. As the observed luminescence not only comes from the condensate containing the vortex but also from non-condensed hot polaritons (our real-space images are the result of the photon collection from $-30^{\circ}$ to $30^{\circ}$ by the numerical aperture 0.5 of our microscope objective), we had to select the condensate emission by spectrally resolving the real-space luminescence image. The spatial distribution of polaritons at the condensate energy is shown in Fig. 2a. The singularity in the spatial phase profile defining the vortex position does indeed correspond to a local minimum of the condensate density, as seen in Fig. 2b. The incomplete reduction of the condensate density at the vortex core is due to diffraction effects that limit the optical resolution of our set-up to about $1 \mu \mathrm{m}$ : our measurements are fully consistent with a strong reduction in density at the vortex core over a diameter smaller than $1 \mu \mathrm{m}$.

A crucial difference of the vortices in the non-equilibrium polariton condensate with respect to standard atomic condensates 

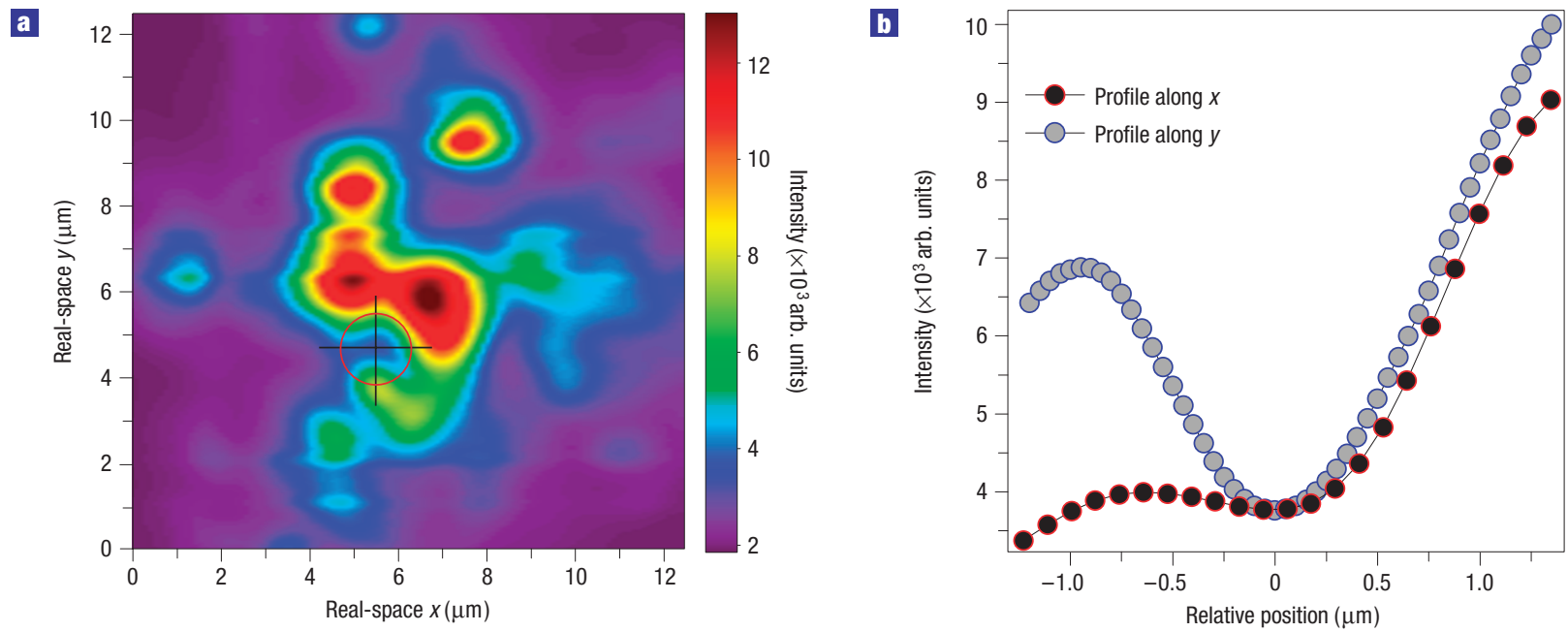

Figure 2 Real-space polariton population at the vortex region. a, Two-dimensional population at the energy of the condensate, over the whole excitation spot, showing the location of the vortex in real space (centre of red circle). The integration in energies was done within the linewidth of the condensate $(\gamma=650 \mu \mathrm{eV})$. $\mathbf{b}$, Population along the two arms of the black cross (black lines in a). The vortex is located at the position where the two lines cross each other, corresponding to a local minimum of the population.
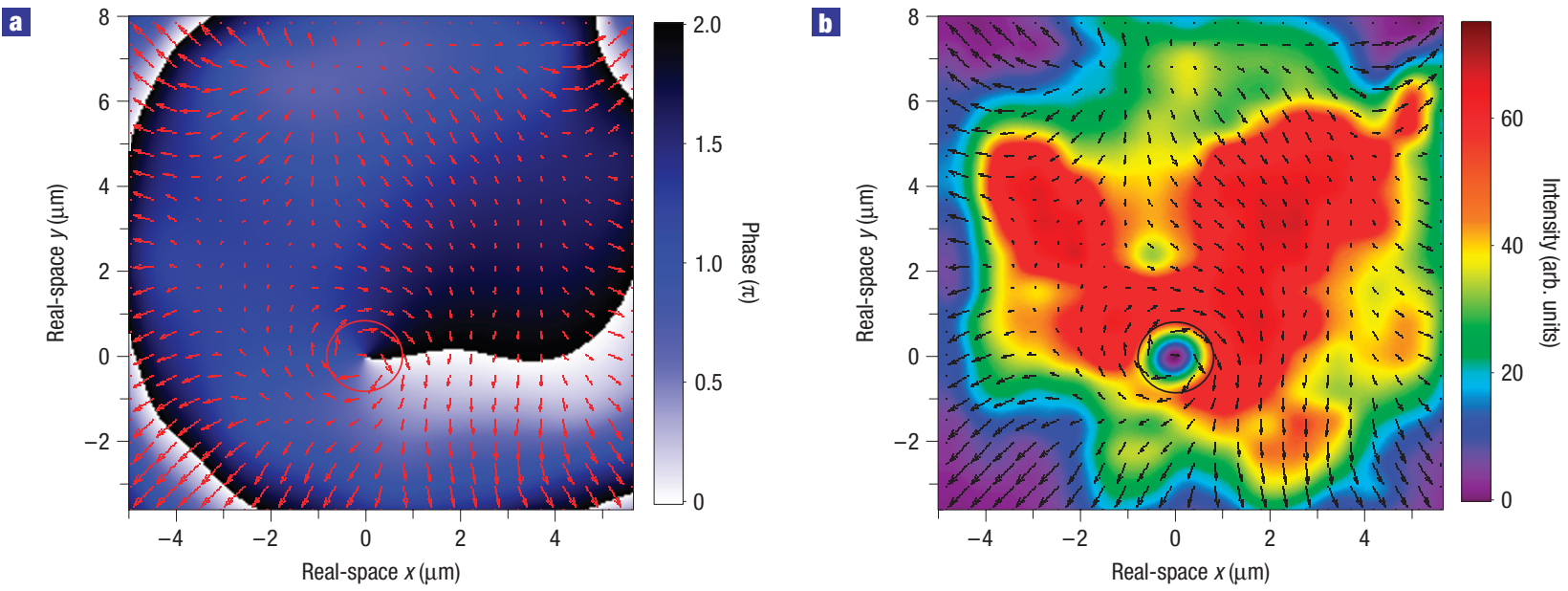

Figure 3 Phase and density distribution from the mean field theory. a, Theoretical phase profile from a simulation with the generalized Gross-Pitaevskii equation in the presence of a disorder potential. The arrows representing the local wavevector $k=\nabla \phi$ wind around the branch point singularity in the phase at the vortex position. $\mathbf{b}$, The real-space density profile drops to zero at the centre of the vortex core.

is the fact that vortices appear spontaneously without the need to stir the condensate. Because of the two-dimensional nature of the polariton condensate, we might naively think of the Berezinskii-Kosterlitz-Thouless phase transition and the spontaneous formation of vortices induced by thermal fluctuations, as recently observed in quasi-two-dimensional ultracold atomic $\mathrm{BECs}^{11,12}$. This mechanism is however contradicted by our experimental observations: as the measured interference patterns result from an average over many runs of the condensate formation, the observed high fringe contrast requires that a vortex of well-defined sign be present at the same position in each run. Although a scenario in which a spontaneously formed vortex is trapped by the disorder could account for a well-defined vortex position, the constancy of its sign is harder to explain. A further argument against the spontaneous proliferation of thermal vortices in the present polariton condensate is the fact that the spatial coherence function (not shown) exhibits a plateau (or a power-law decrease) for large distances, rather than a fast exponential decay as expected for the Berezinskii-Kosterlitz-Thouless phase transition.

The mechanism that is responsible for the spontaneous appearance of the vortex has therefore to be deterministic. The emergence of deterministic flow in polariton non-equilibrium condensates can be attributed to the combined effect of the continuous pumping and inhomogeneity of the system ${ }^{25,26}$ : in marked contrast with equilibrium condensates in which the ground state is always flowless, the presence of pumping and losses makes the condensate polaritons constantly flow down the hills of the disorder potential landscape. Depending on the details of the disorder potential, the non-equilibrium flow pattern may show vortex singularities. A related effect of spontaneous appearance of vortices in a polariton condensate under an external trapping potential was recently discussed in ref. 25 . 
This simple physical picture of the emergence of vortices is supported by numerical simulations based on the mean field model for non-equilibrium condensates recently developed in ref. 27 along the lines of laser theory. Although the phenomenology is in many ways similar to the one observed in lasers ${ }^{19}$, the microscopic physics of the system is however completely different, for example, the nature of the basic bosonic particle under investigation, as well as the condensation kinetic mechanism. An important point to stress in comparison with lasers is that vortices in our polariton $\mathrm{BEC}$ are bound to disorder and have negligible dependence on the excitation conditions, namely excitation power and spot size (see the Supplementary Information).

An example of a simulated image is shown in Fig. 3. A vortex singularity is clearly seen in the density and phase profile. The arrows, which represent the local wavevector $k=\nabla \phi$ (where $\phi$ is the phase), wind around the vortex core. In addition, the vortex core seems to be of the order of $1 \mu \mathrm{m}$, which corresponds approximately to both the characteristic length scale associated with the damping rate $l_{\gamma}=(\hbar / \gamma m)^{1 / 2}$ and the healing length $\xi=\left(\hbar / g|\psi|^{2} m\right)^{1 / 2}$ obtained from the values used in the theoretical simulations. Here $\gamma$ is the damping rate of polaritons, $g$ is the polariton-polariton interaction, $\psi$ is the order parameter and $m$ is the polariton mass. Despite the deterministic nature of the vortex nucleation mechanism, the presence of vortices strongly depends on the position of the excitation laser spot: this experimental observation is recovered by the theoretical simulations, and can be physically understood in terms of the strong dependence of the polariton flow pattern on the disorder.

It would be obviously tempting to consider the observation of persistent quantized vortices in the polariton condensate as a proof of its superfluidity. Unfortunately, no clear-cut criteria for superfluidity in a non-equilibrium system such as ours are yet available. Hence, before using the term superfluidity, a better understanding of the very concept of superfluidity in a non-equilibrium contex $t^{28}$ is needed. It is however safe to conclude that our polariton fluid shares with conventional superfluids such as liquid ${ }^{4} \mathrm{He}$ and Bose-Einstein condensed ultracold atomic gases the crucial property of having a quantized vorticity.

In summary, we have reported on the experimental observation of singly quantized vortices in an exciton-polariton condensate. A clear singularity of $2 \pi$ phase shift has been extracted through interferometric techniques. This quantized phase variation is accompanied by a clear reduction of the polariton fluid density at the vortex location. A theoretical description of the polariton condensate within a mean field model suggests that the vortices arise from the interplay between disorder and the driven-dissipative nature of the polariton condensate.

\section{METHODS}

\section{EXPERIMENTAL REALIZATION}

The experimental set-up is an improved version of the one used in our previous studies $^{3}$. In short, the sample is the same CdTe microcavity cooled down to $4.2 \mathrm{~K}$ by means of a cold-finger liquid-helium flow cryostat. We excited our system in a non-resonant quasicontinuous-wave manner to avoid heating of the sample. The excitation wavelength was chosen in the vicinity of the first minimum of the sample reflection, to efficiently couple light into the cavity. The luminescence of the condensate was observed with a high-numerical-aperture (0.5) microscope objective enabling the achievement of diffraction-limited twodimensional imaging, in conjunction with an interferometric system to acquire the luminescence interferogram. For this purpose, the luminescence was sent in an actively stabilized Michelson interferometer with a mirror-retroreflector configuration (for details see ref. 3) and the real-space interferogram of the luminescence was imaged on a high-resolution CCD (charge-coupled device).

The procedure for the phase extraction of the interferogram is analogous to the one in ref. 29 , where the phase is extracted from the time-frequency domain. Here, the transform is in two dimensions and for real space- $k$ space. The presence of fringes in the luminescence interferogram is of crucial importance because in this way the two conjugate parts of the Fourier transform of the interferogram become separated in the Fourier space. The phase of the interferogram is carried in each of the parts, and by isolating one of them we were able to retrieve the phase information of the interferogram. The retrieved spatial phase profile was then compared with the phase profile of an interferogram without phase singularities but of the same fringe spacing. This difference reveals any phase anomalies or singularities carried in the interferogram.

Spectrally resolved imaging was carried out on the same position of the sample by imaging the real-space luminescence on the entrance slits of the $\sim 10 \mu \mathrm{eV}$ resolution double monochromator. The spectrally resolved real space is then reconstructed from a sufficient number $(\sim 120)$ of spectrally resolved real-space lines. In that way, we were able to extract information on the spatial density profile at the energy of the condensate.

\section{THEORETICAL DESCRIPTION}

The theoretical model that is used to explain the spontaneous appearance of deterministic vortices was introduced in ref. 27; a related method was proposed and applied to polariton condensates containing a vortex in ref. 25. Our mean field model is based on a generalized Gross-Pitaevskii equation for the low-energy polariton states

$$
\begin{aligned}
i \frac{\partial}{\partial t} \psi(\mathbf{r}, t)= & \left\{-\frac{\hbar \nabla^{2}}{2 m}+V_{d}(\mathbf{r})-\frac{i}{2}\left[\gamma_{c}-R\left(n_{\mathrm{R}}(\mathbf{r}, t)\right)\right]\right. \\
& \left.+g|\psi(\mathbf{r}, t)|^{2}+g_{\mathrm{R}} n_{\mathrm{R}}(\mathbf{r}, t)\right\} \psi(\mathbf{r}, t)
\end{aligned}
$$

that takes into account the dissipation of polaritons at a rate $\gamma_{c}$ and their replenishing by stimulated scattering from the exciton reservoir at a rate $R\left(n_{\mathrm{R}}\right)$ that is a function of the exciton reservoir density $n_{\mathrm{R}}$. The photonic disorder is modelled by the potential term $V_{d}$. It is extracted from spatially and energetically resolved photoluminescence measurements far below the condensation threshold. In a first approximation, the lowest emission energy in the luminescence spectrum at a given position corresponds to the local potential energy. Elastic interactions between the condensate polaritons among themselves cause a blueshift of the condensate energy by $g|\psi|^{2}$ and interactions of the condensate polaritons with the reservoir excitons by an amount $g_{\mathrm{R}} n_{\mathrm{R}}$. The equation for the condensate field has to be coupled to a motion equation for the reservoir density. As a simple model, we adopt a rate equation of the form $(\partial / \partial t) n_{\mathrm{R}}(\mathbf{r}, t)=P(\mathbf{r}, t)-\gamma_{\mathrm{R}} n_{\mathrm{R}}(\mathbf{r}, t)-R\left(n_{\mathrm{R}}(\mathbf{r}, t)\right)|\psi(\mathbf{r}, t)|^{2}$ that describes the balance between the pumping rate $P$, the loss from the reservoir and stimulated scattering into the condensate states. This model was shown to recover the elementary excitation spectrum of non-equilibrium condensates predicted by the Keldysh Green function method ${ }^{30}$, as well as the main experimental spatial and spectral features of polariton condensates pumped with a finite excitation spot ${ }^{26}$. The parameters in the equations are phenomenological and should in principle be determined from the experiment. Unfortunately, insufficient experimental data are available to univocally determine them. The parameters that were used in the simulations were: $m \hbar^{-2}=1.7 \mathrm{meV}^{-1} \mu \mathrm{m}^{-2}, \hbar \gamma=1 \mathrm{meV}, \hbar \gamma_{\mathrm{R}}=10 \mathrm{meV}, \hbar g=0.04 \mathrm{meV}_{\mu} \mathrm{m}^{2}$,

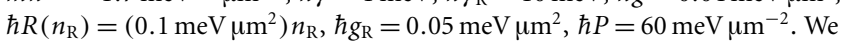
have however verified that the possibility of observing vortices does not depend on the precise values of the model parameters.

Received 16 January 2008; accepted 7 July 2008; published 10 August 2008.

References

1. Donnelly, R. J. Quantized Vortices in Helium II (Cambridge Univ. Press, Cambridge, 1991).

2. Pitaevskii, L. P. \& Stringari, S. Bose-Einstein Condensation (Clarendon, Oxford, 2003).

3. Kasprzak, J. et al. Bose-Einstein condensation of exciton polaritons. Nature 443, 409-414 (2006).

4. Christopoulos, S. et al. Room-temperature polariton lasing in semiconductor microcavities. Phys. Rev. Lett. 98, 126405 (2007).

5. Balili, R. et al. Bose-Einstein condensation of microcavity polaritons in a trap. Science 316, 1007-1010 (2007).

6. Bajoni, D. et al. Polariton laser using single micropillar GaAs-GaAlAs semiconductor cavities. Phys. Rev. Lett. 100, 047401 (2008).

7. Fetter, A. L. \& Svidzinsky, A. A. Vortices in trapped dilute Bose-Einstein condensate. J. Phys. Condens. Matter 13, R135-R194 (2001).

8. Matthews, M. R. et al. Vortices in a Bose-Einstein condensate. Phys. Rev. Lett. 83, 2498-2501 (1999).

9. Madison, K. W. et al. Vortex formation in a stirred Bose-Einstein condensate. Phys. Rev. Lett. 84, $806-809$ (2000). 
10. Inouye, S. et al. Observation of vortex phase singularities in Bose-Einstein condensates. Phys. Rev. Lett. 87, 080402 (2001)

11. Simula, T. P. \& Blakie, P. B. Thermal activation of vortex-antivortex pairs in quasi-two-dimensional Bose-Einstein condensates. Phys. Rev. Lett. 96, 020404 (2006).

12. Giorgetti, L., Carusotto, I. \& Castin, Y. Semiclassical field method for the equilibrium Bose gas and application to thermal vortices in two dimensions. Phys. Rev. A 76, 013613 (2007).

13. Hadzibabic, Z. et al. Berezinskii-Kosterlitz-Thouless crossover in a trapped atomic gas. Nature 441, 1118-1121 (2006).

14. Schweikhard, V., Tung, S. \& Cornell, E. A. Vortex proliferation in the Berezinskii-Kosterlitz-Thouless regime on a two-dimensional lattice of Bose-Einstein condensates. Phys. Rev. Lett. 99, 030401 (2007)

15. Minnhagen, $P$. The two-dimensional Coulomb gas, vortex unbinding, and superfluid-superconducting films. Rev. Mod. Phys. 59, 1001-1066 (1987).

16. Richard, M. et al. Experimental evidence for nonequilibrium Bose condensation of exciton polaritons. Phys. Rev. B 72, 201301(R) (2005).

17. Baas, A. et al. Synchronized and desynchronized phases of exciton-polariton condensates in the presence of disorder. Phys. Rev. Lett. 100, 170401 (2008).

18. Savona, V. et al. Optical properties of microcavity polaritons. Phase Transit. 68, 169-279 (1999).

19. Scheuer, J. et al. Optical vortices crystals: Spontaneous generation in nonlinear semiconductor microcavities. Science 285, 230-233 (1999).

20. Chen, Y. F. \& Lan, Y. P. Formation of optical vortex lattices in solid-state microchip lasers: Spontaneous transverse mode locking. Phys. Rev. A 64, 063807 (2001).

21. Vaupel, M. \& Weiss, C. O. Circling optical vortices. Phys. Rev. A 51, 4078-4085 (1995).

22. Bolda, E. L. et al. Detection of vorticity in Bose-Einstein condensed gases by matter-wave interference. Phys. Rev. Lett. 81, 5477-5480 (1998).

23. Tempere, J. et al. Fringe pattern of interfering Bose-Einstein condensates with a vortex. Solid State Commun. 108, 993-996 (1998).
24. Andrews, M. R. et al. Observation of interference between two Bose condensates. Science 275, 637-641 (1997)

25. Keeling, J. \& Berloff, N. G. Spontaneous rotating vortex lattices in a pumped decaying condensate. Phys. Rev. Lett. 100, 250401 (2007).

26. Wouters, M., Carusotto, I. \& Ciuti, C. Spatial and spectral shape of inhomogeneous non-equilibrium exciton-polariton condensates. Phys. Rev. B 77, 115340 (2008).

27. Wouters, M. \& Carusotto, I. Excitations in a nonequilibrium Bose-Einstein condensate of exciton polaritons. Phys. Rev. Lett. 99, 140402 (2007).

28. Wouters, M. \& Carusotto, I. Excitations and superfluidity in non-equilibrium Bose-Einstein condensates of exciton-polaritons. Preprint at <http://arxiv.org/abs/0707.1446> (2007).

29. Langbein, W. et al. Microscopic measurement of photon echo formation in groups of individual excitonic transitions. Phys. Rev. Lett. 95, 017403 (2005).

30. Szymańska, M. H., Keeling, J. \& Littlewood, P. B. Nonequilibrium quantum condensation in an incoherently pumped dissipative system. Phys. Rev. Lett. 96, 230602 (2006).

Supplementary Information accompanies this paper on www.nature.com/naturephysics.

\section{Acknowledgements}

We thank D. Sarchi, V. Savona, B. Pietka, J. Tempere and J. Devreese for fruitful discussions. The work was supported by the Swiss National Research Foundation through 'NCCR Quantum Photonics'.

\section{Author information}

Reprints and permission information is available online at http://npg.nature.com/reprintsandpermissions. Correspondence and requests for materials should be addressed to K.G.L or M.W. 